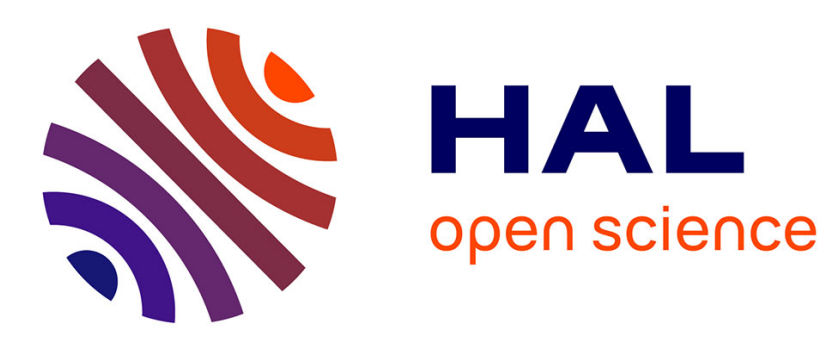

\title{
Structure et enregistrement des traces latentes d'ions argon et fer dans l'olivine et le mica muscovite
}

\author{
E. Dartyge, M. Lambert, M. Maurette
}

\section{To cite this version:}

E. Dartyge, M. Lambert, M. Maurette. Structure et enregistrement des traces latentes d'ions argon et fer dans l'olivine et le mica muscovite. Journal de Physique, 1976, 37 (7-8), pp.939-943. 10.1051/jphys:01976003707-8093900 . jpa-00208489

\section{HAL Id: jpa-00208489 https://hal.science/jpa-00208489}

Submitted on 1 Jan 1976

HAL is a multi-disciplinary open access archive for the deposit and dissemination of scientific research documents, whether they are published or not. The documents may come from teaching and research institutions in France or abroad, or from public or private research centers.
L'archive ouverte pluridisciplinaire HAL, est destinée au dépôt et à la diffusion de documents scientifiques de niveau recherche, publiés ou non, émanant des établissements d'enseignement et de recherche français ou étrangers, des laboratoires publics ou privés. 


\title{
STRUCTURE ET ENREGISTREMENT DES TRACES LATENTES D'IONS ARGON ET FER DANS L'OLIVINE ET LE MICA MUSCOVITE
}

\author{
E. DARTYGE, M. LAMBERT \\ Laboratoire de Physique des Solides (*), Bâtiment 510, Université Paris-Sud, \\ 91405 Orsay, France
}

et

M. MAURETTE

Laboratoire René-Bernas, 91405 Orsay, France

(Reçu le 12 février 1976, accepté le $1^{\mathrm{er}}$ mars 1976)

\begin{abstract}
Résumé. - On étudie, par diffusion des rayons $\mathrm{X}$ aux petits angles, la nature de la trace latente des ions $\mathrm{Fe}$ et $\mathrm{Ar}$ (énergie comprise entre 1 et $7 \mathrm{MeV} /$ nucléon) dans l'olivine et le mica muscovite. Dans les deux cas, il se forme des zones endommagées qu'on caractérise par leur taille et leur espacement. La distance inter-zones conditionne la mise en évidence des traces par attaque chimique : l'enregistrement des traces n'apparaît pas lié à un mécanisme à seuil énergétique.
\end{abstract}

Abstract. - X-ray small angle scattering experiments are used to study the latent tracks produced by $\mathrm{Fe}$ and $\mathrm{Ar}$ ions (energy range : 1-7 MeV/a.m.u.) in olivine and muscovite mica. In both cases, zones of disordered material are formed which we have characterized by their size and interzone distance. This later distance appears to be the relevant parameter for the chemical etching of the latent track : the track registration process does not seem to imply the existence of an energy threshold mechanism.

1. Introduction. - L'étude des traces d'ions lourds dans les isolants a pu se développer après la découverte [1] d'un processus de détection de la trace latente par attaque chimique : la trace attaquée peut alors être observée directement au moyen d'un microscope optique. Après avoir étudié des détecteurs minéraux de sensibilité différente irradiés par des ions de nature différente et d'énergie variable, Price et al. ont ainsi pu définir, pour l'enregistrement des traces attaquées, un seuil critique du taux d'ionisation primaire $J_{\mathrm{C}}$ au-dessous duquel aucune trajectoire d'ion ne peut être révélée par attaque chimique. La valeur de $J_{C}$, déterminée expérimentalement pour chaque détecteur, peut être comparée aux valeurs de la perte d'énergie des ions par ionisation $J$ calculée à partir de la formule établie par Bethe :

$$
J \sim \frac{Z_{\mathrm{e}}^{2}}{\beta^{2}}\left[\log \frac{\beta^{2}}{1-\beta^{2}}+K-\beta^{2}\right]
$$

$\left(^{*}\right)$ Laboratoire associé au C.N.R.S.
$Z_{\mathrm{e}}$ étant la charge effective de l'ion, $\beta$ le rapport de sa vitesse à la vitesse de la lumière, $K$ une constante caractéristique du détecteur.

Nous avons reporté figure 1 les courbes donnant les variations de $J$ en fonction de l'énergie de l'ion incident et ceci pour 2 ions, $\mathrm{Fe}$ et $\mathrm{Ar}$, et pour les deux détecteurs minéraux que sont l'olivine et le mica. Nous avons utilisé pour cela les valeurs de $K$ égales respectivement à 9,7 et 14,2 déterminées expérimentalement par Price et al. [2]. Il est certain que les valeurs de $J$ ainsi calculées ne sont correctes que dans le domaine des hautes énergies; à basse énergie, le taux d'ionisation décroît : $J$ passe par une valeur maximale pour une énergie des ions de l'ordre de $1 \mathrm{MeV} /$ nucléon. En comparant la valeur de $J_{\mathrm{C}}$ aux valeurs calculées de $J$, on peut constater figure 1 que les deux cristaux ont un comportement différent en ce qui concerne la détection des traces attaquées :

i) MicA. - Les ions argon ne sont efficaces que pour une énergie inférieure à $3 \mathrm{MeV} /$ nucléon alors que les ions $\mathrm{Fe}$ sont efficaces dans tout le domaine d'énergie considéré. 
ii) Olivine. - Les ions argon ne produisent pas de traces détectées par attaque chimique alors que les ions $\mathrm{Fe}$ sont efficaces pour une énergie inférieure à $3 \mathrm{MeV} /$ nucléon.
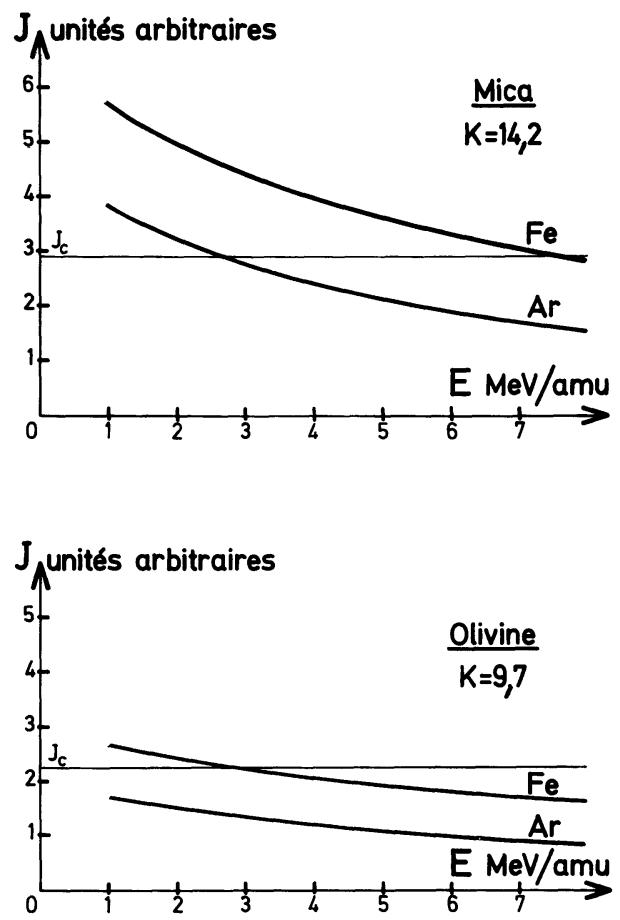

FIG. 1. - Taux d'ionisation en fonction de la vitesse des ions $\mathrm{Fe}$ et Ar dans l'olivine et le mica muscovite, calculés d'après la référence [2]. Les seuils approximatifs $J_{\mathrm{C}}$ de l'enregistrement des traces par le mica et l'olivine sont représentés.

L'existence du taux critique d'ionisation $J_{\mathrm{C}}$, déterminé par l'observation des traces attaquées, pourrait être liée à une structure caractéristique de la trace latente : en effet, au cours d'expériences précédentes [3], nous avons montré que, lorsque la trace latente est susceptible d'être révélée chimiquement, elle consiste, en fait, en une région du cristal renfermant des défauts de taille relativement grande (diamètre de $22 \AA$ pour les ions néon, $40 \AA$ pour les ions argon et plus de $60 \AA$ pour les ions krypton), ces défauts sont assez proches les uns des autres et vraisemblablement constitués de matière amorphe. Bien plus, dans le cas de l'irradiation du mica par ions Ar d'énergie variable (1-10 MeV/nucléon) [4], nous n'avons pas alors détecté de défauts étendus créés par des ions d'énergie supérieure à $5 \mathrm{MeV} /$ nucléon. Cependant, nous avions noté une diminution progressive du nombre de défauts créés avec l'énergie de l'ion incident, contrairement à une disparition brutale de ces défauts lorsque le taux d'ionisation primaire dans le détecteur est inférieur à $J_{\mathrm{C}}$.

Nous avons donc repris l'étude de la trace latente pour les deux détecteurs, de structure et de sensibilité (pour la trace attaquée) différentes, que sont l'olivine et le mica. Cette étude a pour but :

- de vérifier la généralité de nos premières observations relatives à la structure de la trace latente, en particulier par l'observation des échantillons irradiés aux ions $\mathrm{Fe}$;

- de déterminer, en utilisant des doses élevées pour des irradiations telles que $J<J_{\mathrm{C}}$, si le taux critique d'ionisation $J_{\mathrm{C}}$ intervient pour la formation des défauts étendus présents dans la trace latente ou seulement dans la transformation de cette trace par attaque chimique.

2. Dispositifs expérimentaux et description des échantillons. - Nous avons donc étudié des échantillons monocristallins d'olivine et de mica irradiés par des ions $\mathrm{Fe}$ et Ar d'énergies incidentes respectives 7,2 et 7,5 MeV/nucléon. Les irradiations ont été effectuées à l'aide de l'accélérateur A.L.I.C.E. d'Orsay; le dispositif d'irradiation a été décrit par ailleurs [4]. Les échantillons, sous forme de lamelles très minces, étaient placés perpendiculairement au faisceau d'ions incidents. Dans le cas du mica, pour lequel on peut obtenir facilement des lamelles de très faible épaisseur $(10 \mu)$, l'irradiation par des ions d'énergie variable (7 à 1,3 MeV/nucléon) était réalisée par empilement de lamelles d'épaisseurs calibrées, la traversée de l'échantillon suffit à diminuer progressivement l'énergie des ions. Celle-ci n'est plus aussi bien définie que celle des ions incidents mais suffisamment précise pour nos mesures. Dans le cas de l'olivine, on ne peut pas disposer de lamelles aussi minces ; nous avons donc irradié des lamelles d'épaisseurs différentes $(30 \mu$ et $60 \mu$ ) et nous avons obtenu ainsi des échantillons irradiés, les uns par des ions d'énergie élevée, les autres par des ions dont l'énergie prenait toutes les valeurs inférieures à $7 \mathrm{MeV} /$ nucléon. Le tableau I précise les conditions expérimentales pour chaque échantillon. Nous avons calculé la perte d'énergie totale dans chaque échantillon de mica muscovite et d'olivine ainsi que le parcours des ions Fe de 7,2 MeV/nucléon à l'aide des tables de Henke et Benton [5].

Ces échantillons ont ensuite été étudiés par diffusion des rayons $X$ aux petits angles en utilisant le même dispositif décrit précédemment [4]. Le rayonnement est monochromatique $(\mathrm{CuK} \alpha)$, de longueur d'onde $\lambda=1,54 \AA$, et nous mesurons le pouvoir diffusant $I(s)$ de l'échantillon pour un vecteur de diffusion de longueur $S=\frac{2 \sin \theta}{\lambda}, 2 \theta$ étant l'angle de diffusion.

Le pouvoir diffusant $I(s)$ représente le nombre d'électrons qui, diffusant les rayons $\mathrm{X}$ indépendamment, donneraient la même intensité que celle correspondant à une unité de volume d'échantillon. Rappelons ici qu'à des défauts ponctuels correspond un pouvoir diffusant indépendant de l'angle de diffusion et que des défauts étendus donnent lieu à un pouvoir diffusant décroissant lorsque $s$ croît. L'analyse des courbes de diffusion permet de déterminer la taille des régions endommagées. Quant à leur nombre, sa détermination absolue dépend d'une hypothèse sur la densité électronique, c'est-à-dire sur la nature même de ces zones [6]. 
TABLEAU I

\begin{tabular}{|c|c|c|c|c|c|c|c|c|c|c|}
\hline \multirow{3}{*}{$\begin{array}{c}\text { Echantillon } \\
-\end{array}$} & \multirow{3}{*}{$\begin{array}{c}\text { Epaisseur }=e \\
\qquad \begin{array}{c}(\mu) \\
\frac{-}{30}\end{array}\end{array}$} & \multicolumn{2}{|c|}{$\begin{array}{c}\text { Nature } \\
\text { de l'ion } \\
\text { et parcours } \\
(\mu)\end{array}$} & \multirow{3}{*}{$\begin{array}{c}\text { Variation-Energie } \\
\text { dans échantillon } \\
\text { (MeV/nucléon) } \\
-\overline{7,5-4,5}\end{array}$} & \multicolumn{2}{|c|}{$\begin{array}{c}\text { Dose }=\Phi \\
\text { Particules } / \mathrm{cm}^{2}\end{array}$} & \multirow{3}{*}{$\begin{array}{l}\text { Diamètre } \\
\text { des défauts } \\
\qquad \begin{array}{c}(\AA) \\
- \\
32\end{array}\end{array}$} & \multirow{3}{*}{$\begin{array}{c}n\left(\frac{\delta \rho}{\rho_{0}}\right)^{2} \\
\left(\mu^{-1}\right) \\
- \\
0\end{array}$} & \multirow{3}{*}{$\begin{array}{c}n\left(^{*}\right) \\
\left(\mu^{-1}\right) \\
- \\
0\end{array}$} & \multirow{3}{*}{$\begin{array}{l}\text { Distance } \\
\text { entre défauts } \\
\text { ( }) \\
-\end{array}$} \\
\hline & & & & & & - & & & & \\
\hline & & $\mathrm{Ar}$ & 60 & & 6 & $\times 10^{12}$ & & & & \\
\hline \multirow{3}{*}{ Olivine } & 60 & $\mathrm{Ar}$ & 60 & $7,5-0$ & & $\times 10^{12}$ & 32 & 0,7 & 8 & 1250 \\
\hline & 30 & $\mathrm{Fe}$ & 54 & $7,2-3,7$ & & $\times 10^{12}$ & 40 & 2,1 & 23 & 430 \\
\hline & 60 & $\mathrm{Fe}$ & 54 & $7,2-0$ & 1,4 & $\times 10^{12}$ & 40 & 20 & 222 & 45 \\
\hline \multirow{4}{*}{ Mica } & 8 & $\mathrm{Fe}$ & 54 & $7,2-6,3$ & 5 & $\times 10^{11}$ & 46 & 0,84 & 37 & 270 \\
\hline & 17 & $\mathrm{Fe}$ & 54 & $3,8-1,3$ & 5 & $\times 10^{11}$ & 46 & 1,87 & 81 & 123 \\
\hline & 8 & $\mathrm{Fe}$ & 54 & $1,3-0$ & 5 & $\times 10^{11}$ & 46 & 5,6 & 243 & 42 \\
\hline & 20 & $\mathrm{Ar}$ & 60 & $7,5-5,75$ & 7 & $\times 10^{12}$ & 32 & 0,27 & 12 & 833 \\
\hline
\end{tabular}

(*) Calculé en prenant $\frac{\delta \rho}{\rho_{0}}=0,15$ (mica) 0,30 (olivine).

3. Résultats expérimentaux. - Les résultats des mesures de diffusion de rayons $X$ aux petits angles sont représentés figures 2 et 3 . On constate que dans tous les cas (sauf pour l'irradiation de l'olivine avec des ions Ar de haute énergie, $E>3,7 \mathrm{MeV} /$ nucléon; échantillon d'épaisseur $30 \mu$, figure $2 c$ ), les courbes sont caractéristiques de la présence de défauts étendus :

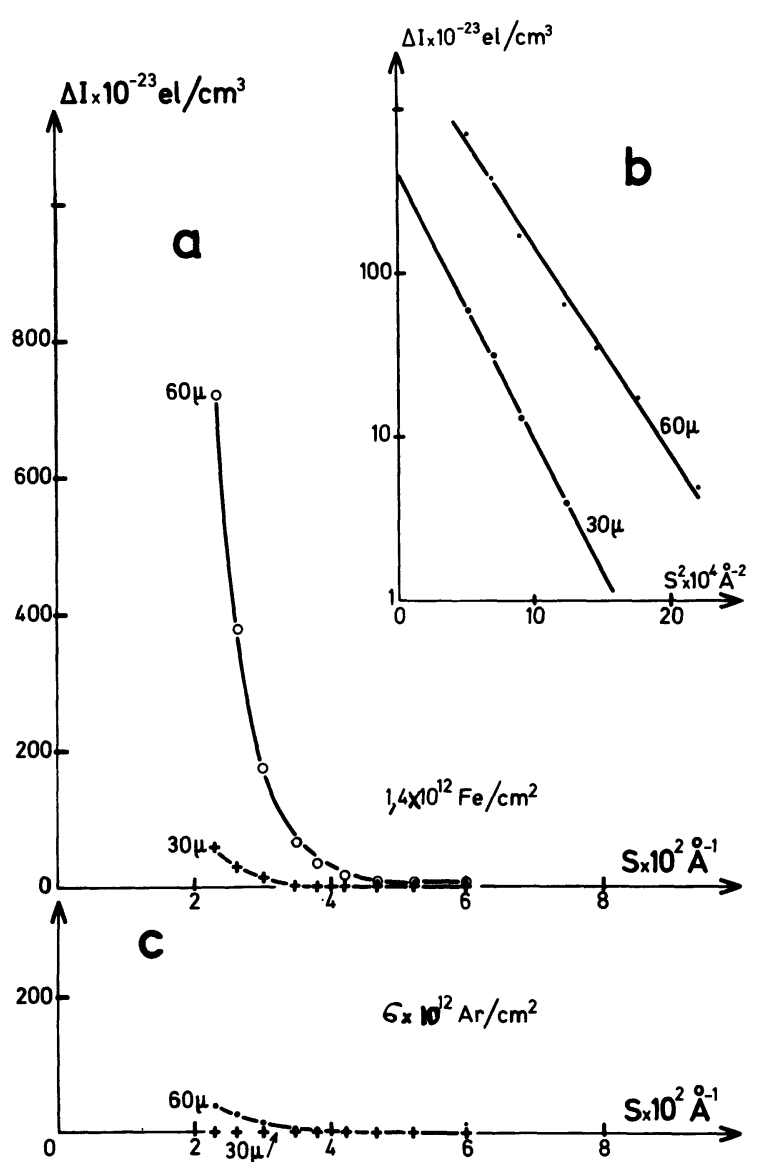

Fig. 2. - Pouvoir diffusant $\Delta I$ de cristaux d'olivine de 30 et $60 \mu$ d'épaisseur par des ions de fer et d'argon. Le pouvoir diffusant de l'échantillon non irradié est soustrait. a) Irradiation par des ions de fer de 7,2 MeV/nucléon. $b$ ) Analyse en gaussienne de la courbe a) $\Delta I(s)=\Delta I(0) \exp \left(-\frac{4 \pi^{2} R^{2} s^{2}}{5}\right), R$ rayon des défauts [7]. c) Irradiation par des ions argon de 7,5 $\mathrm{MeV} /$ nucléon.

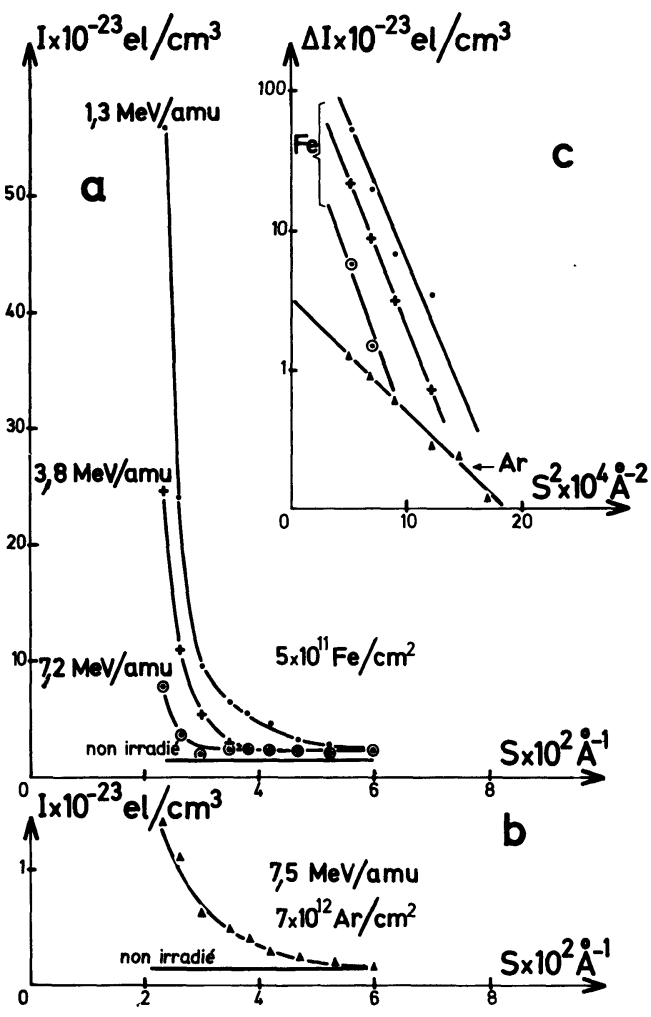

Fig. 3. - a) Pouvoir diffusant $I$ de cristaux de mica muscovite irradiés par des ions de fer. $b$ ) Pouvoir diffusant $I$ de cristaux de mica muscovite irradiés par des ions argon. c) Analyse en gaussienne des courbes $a$ ) et $b) \Delta I(s)=\Delta I(0) \exp \left(-\frac{4 \pi^{2} R^{2} s^{2}}{5}\right), R$ rayon des défauts [7]. La contribution des défauts ponctuels à la diffusion a été soustraite.

donc, il y a toujours dans les échantillons irradiés formation de défauts de grande taille.

D'autre part, il est possible d'analyser les courbes de diffusion en gaussienne [6] (Fig. $2 b$ et $3 c$ ), ce qui indique que, dans chaque échantillon, les défauts ont une taille uniforme. Le diamètre $2 R$ des défauts est donné directement par la mesure de la pente des droites (Fig. $2 b$ et $3 c$ ). On constate que pour un détecteur donné la pente ne varie sensiblement pas avec l'énergie 
des ions incidents : la taille des défauts est indépendante de l'énergie de l'ion incident, mais change avec le numéro atomique de l'ion.

3.1 Olivine. - Les défauts induits par les ions fer ont un diamètre de $40 \AA$ quelle que soit leur énergie comprise entre 7 et $1 \mathrm{MeV} /$ nucléon. Cependant, les ions efficaces sont ceux de basse énergie, puisque l'échantillon le plus épais renferme environ 10 fois plus de zones endommagées (par unité de volume) que l'échantillon le plus mince.

Les défauts induits par les ions argon ont un diamètre de $32 \AA$. On ne les observe que dans l'échantillon le plus épais : ils sont donc formés par des ions d'énergie inférieure à $3,7 \mathrm{MeV} /$ nucléon. L'efficacité d'endommagement est dans ce cas 20 fois moins grande que dans le cas des ions Fe de basse énergie. Il est possible qu'une dose d'irradiation plus élevée permette d'observer également ces mêmes défauts dans le cas d'irradiation à haute énergie.

3.2 Mica muscovite. - Dans le cas de l'irradiation par des ions $\mathrm{Ar}$, des résultats précédents [5], obtenus pour des ions d'énergies variant entre 10 et $1 \mathrm{MeV} /$ nucléon, avaient montré la formation de défauts de $40 \AA$ de diamètre et ceci uniquement pour une énergie des ions incidents inférieure ou égale à $5 \mathrm{MeV} /$ nucléon. Remarquons cependant que ces résultats n'excluaient pas la formation de défauts en faible concentration pour des ions d'énergie plus grande.

Nous pouvons constater, figure $2 b$, que ces défauts existent également à haute énergie; ils ont un diamètre légèrement inférieur à ceux créé à basse énergie ( $32 \AA$ au lieu de $40 \AA$ ) et sont obtenus avec un rendement très faible (trois fois plus faible que les ions $\mathrm{Fe}$ de même énergie, en tenant compte des doses d'irradiation différentes.

Dans le cas d'irradiation avec des ions $\mathrm{Fe}$, les défauts ont le même diamètre $(\sim 46 \AA)$ pour toutes les énergies considérées. Leur concentration diminue d'un facteur 7 lorsque l'énergie des ions augmente de 1,3 à $7,2 \mathrm{MeV} /$ nucléon.

Dans toutes ces expériences, nous nous sommes limités à une dose d'irradiation de $5 \times 10^{11}$ ions $\mathrm{Fe} / \mathrm{cm}^{2}$. En effet, comme l'efficacité des ions $\mathrm{Fe}$ est grande, il apparaît aux énergies inférieures ou égales à $3 \mathrm{MeV} /$ nucléon une saturation dans le nombre des défauts créés qui est due au recouvrement des zones endommagées pour des doses supérieures à $10^{12}$ ions $\mathrm{Fe} / \mathrm{cm}^{2}$ (dans le cas des ions argon, les mêmes effets se produisent, mais pour des doses supérieures à $10^{13}$ ions $/ \mathrm{cm}^{2}$ ). Cette saturation coïncide avec l'apparition d'une phase amorphe pouvant être observée par diffraction des rayons X [3]. Dans l'olivine, ce phénomène d'amorphisation apparaît également, mais pour des doses d'ions de fer 10 fois plus élevées et pour des énergies inférieures à $3,7 \mathrm{MeV} /$ nucléon.
4. Discussion. - 4.1 EnRegistrement des traces LATENTES D'IONS LOURDS DANS LES SOLIDES ISOLANTS. Dans l'olivine et le mica, la trace latente des ions Ar et $\mathrm{Fe}$ est donc constituée par des défauts étendus. Leur taille, pour un détecteur donné, dépend en fait fortement du numéro atomique $Z$ de l'ion incident : dans le cas du mica, elle varie de 22 à $60 \AA$ lorsque $Z$ passe de 10 à 36 [3]; dans le cas de l'olivine, elle varie de 32 à $40 \AA$, lorsque $Z$ passe de 18 à 26 .

Ces hétérogénéités de densité électronique sont présentes dans les deux détecteurs dès l'irradiation et leur caractérisation est importante pour la discussion du mécanisme de formation des traces. Si leur taille est facile à mesurer, il est cependant difficile de préciser leur structure, puisque nous n'avons aucune information directe. Les seuls renseignements disponibles sont les suivants :

i) dans les deux cas, une irradiation prolongée transforme le cristal en solide amorphe : les défauts sont donc certainement constitués de matière désorganisée. L'hypothèse la plus simple est que ces défauts sont des îlots amorphes dans un solide cristallin ;

ii) nous pouvons connaître, pour chaque échantillon, le produit de la concentration de ces défauts $N$ par le carré de leur différence de densité électronique, $\delta \rho$, avec le reste du cristal. En effet, si $\Delta I(0)$ est le pouvoir diffusant de l'échantillon obtenu par extrapolation, à l'angle de diffusion nul, de la courbe $\Delta I(s)$ (Fig. $2 b$ et $3 b$ ) et si $V$ est le volume des défauts :

$$
\Delta I(0)=N \delta \rho^{2} V^{2} .
$$

Nous avons reporté (tableau I) les valeurs de $n\left(\frac{\delta \rho}{\rho_{0}}\right)^{2}, \rho_{0}$ étant la densité électronique du cristal, $n$ étant le nombre de défauts créé par ion incident et par unité de longueur $(\mu)$ de trajectoire.

Dans un travail précédent [3], nous avions évalué la variation relative de densité $\frac{\delta \rho}{\rho_{0}}$ à $5 \%$, par analogie avec la variation de densité du quartz rendu amorphe par irradiation aux neutrons rapides [7]. Ceci nous avait permis de déterminer, pour le mica, des valeurs à la fois de la concentration des défauts et de leur distance le long de la trajectoire des ions. Nous ne disposions alors que d'une seule énergie pour les ions incidents et n'avions aucune possibilité de vérifier cette hypothèse. Dans le cas présent, une variation de $5 \%$ de la densité n'est pas compatible avec les résultats obtenus pour tous les échantillons de mica : en particulier, dans le cas de l'irradiation à basse énergie (1,3-0 MeV/nucléon), on obtiendrait alors une distance entre défauts de quelques $\AA$, ce qui est impossible. Nous avons donc fait figurer, tableau I, les valeurs du nombre $n$ de défauts créés par unité de longueur $(\mu)$ et par ion incident, et de la distance $d$ entre défauts le long de la trajectoire des ions calculées pour une valeur arbitraire $\frac{\delta \rho}{\rho_{0}}=15 \%$. Ceci conduit à 
des valeurs de $d$ comprises entre 46 et $360 \AA$, ces valeurs seront discutées dans le paragraphe suivant.

Dans le cas des échantillons d'olivine, nous avons de même évalué $n$ et $d$ pour une variation relative de densité entre défauts et matrice de $30 \%$. Ces valeurs de $n$ et de $d$ sont donc données à titre indicatif, seule la valeur de $n\left(\frac{\delta \rho}{\rho_{0}}\right)^{2}$ est une donnée expérimentale. Si on admet que $\frac{\delta \rho}{\rho_{0}}$ est une constante caractéristique du cristal considéré et ne varie pas en cours d'irradiation, les valeurs de $n\left(\frac{\delta \rho}{\rho_{0}}\right)^{2}$ sont inversement proportionnelles à $d$. Nous voyons donc que, pour les 2 types de détecteurs, le taux de création de défauts croît très vite lorsque l'énergie des ions incidents diminue : il est multiplié par 7 dans le cas du mica pour une énergie des ions Fe incidents passant de 7 à $1,3 \mathrm{MeV} /$ nucléon. Si nous nous reportons aux courbes de pertes d'énergie par ionisation par unité de longueur de trajectoire (Fig. 1), nous constatons que la variation de $J$ est beaucoup plus faible dans la même gamme d'énergies. Nos résultats montrent donc que la formation des gros défauts n'est pas reliée par une loi de proportionnalité simple au taux d'ionisation dans le détecteur.

D'autre part, nous n'observons pas de changement de nature des défauts formés lorsque l'énergie des ions devient plus grande (supérieure à la valeur correspondant au seuil de détection indiqué sur la figure 1). On observe simplement une diminution progressive du taux de formation des défauts avec l'augmentation d'énergie des ions incidents.

4.2 ENREGISTREMENT DES TRACES RÉVÉLÉES PAR ATTAQUE CHIMIQUE. - Si l'on se reporte à la figure 1, on constate que le modèle de Fleischer et al. prévoit que, dans l'olivine, les ions Ar à toutes énergies et les ions fer d'énergie supérieure à $3 \mathrm{MeV} /$ nucléon ne peuvent former de trace attaquable. Dans le cas du mica, le seuil critique se trouverait aux environs de $8 \mathrm{MeV} /$ nucléon pour le fer. Cependant, nous venons de montrer qu'il n'y a aucune discontinuité de la nature des défauts qui composent la trace latente de part et d'autre des seuils prédits par Fleischer et al. Nous pensons donc que la formation de la trace attaquée n'est pas liée à un mécanisme à seuil, mais reflète simplement l'existence d'un espacement minimum entre les îlots de dégâts successifs permettant l'attaque chimique rapide et continue de la trace latente. $\mathrm{Ce}$ résultat est en accord avec des expériences de recuits thermiques partiels de traces latentes [8] suivies d'observation au microscope électronique : les traces latentes partiellement recuites apparaissent au microscope électronique sous forme de lignes de contraste discontinues et ne sont plus attaquables lorsque la distance entre îlots de dégâts successifs est de l'ordre de $100 \AA$. Si nous nous reportons au tableau I, les valeurs de $\frac{\delta \rho}{\rho_{0}}$ ont été fixées pour obtenir le même ordre de grandeur pour l'espacement des défauts lorsque la trace est révélable par attaque chimique.

5. Conclusion. - En conclusion, en étendant nos expériences à un détecteur minéral d'ions lourds de structure et sensibilité très différentes de celles du mica muscovite, nous avons vérifié que les traces d'ions lourds dans les solides isolants présentent les caractéristiques générales suivantes : elles sont constituées de zones désordonnées ou amorphes, de diamètre variant entre 22 et $60 \AA$ suivant le numéro atomique de l'ion, l'espacement de ces zones à haute énergie augmente avec l'énergie de l'ion incident, ce qui rend la trace de plus en plus difficile à attaquer. Nous insistons sur le fait qu'il n'y a pas de discontinuité dans la nature de la trace latente de part et d'autre du seuil d'ionisation critique proposé par Fleischer et al., ce qui implique d'après nous qu'il n'existe pas de mécanisme à seuil pour la formation de la trace latente.

Nous avons, dans le cadre de cet article, comparé nos résultats uniquement aux prédictions de la théorie de Price et al., qui est la plus adoptée pour les détecteurs minéraux. Cependant, en étendant nos mesures à des domaines d'énergie plus étendus, en particulier à basse énergie $(\simeq 0,2 \mathrm{MeV} /$ nucléon), nous espérons comparer le taux de création des défauts étendus à des évaluations des sections efficaces des différents mécanismes d'interactions ions lourds-matière (cascades de déplacements ou bien excitation des couches internes et ionisation multiple, par exemple) et trouver ainsi le mécanisme dominant pour la formation de la trace latente dans les minéraux.

Remerciements. - Nous remercions L. Deschamps pour sa participation technique aux expériences et J. P. Duraud, les équipes des accélérateurs L.I.N.A.C. de Manchester et A.L.I.C.E. d'Orsay pour les irradiations.

\section{Bibliographie}

[1] Fleischer, R. L., Price, P. B. and Walker, R. M., J. Appl. Phys. 36 (1965) 3645

[2] Price, P. B., Fleischer, R. L. and Moak, C. D., Phys. Rev. 167 (1968) 277.

[3] Dartyge, E., Lambert, M., Radiat. Eff. 21 (1974) 71.

[4] Lambert, M., Levelut, A.-M., Maurette, M. and HeckMANN, J., Radiat. Eff. 3 (1970) 155.
[5] HenKe, R. P. and Benton, E. V., USNRDLTR 1102 (1966) 21. [6] GuINIER, A., Théorie et Technique de Radiocristallographie (Dunod, Ed.) 1963.

[7] COMES, R., LAmbert, M., GuINIER, A., Interaction of radiation with solids (Plenum Press) 1967, p. 319.

[8] Maurette, M., J. Physique 27 (1966) 505. 